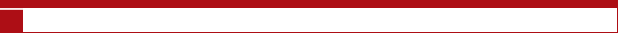

SECCIÓN: Informes y avances de investigación
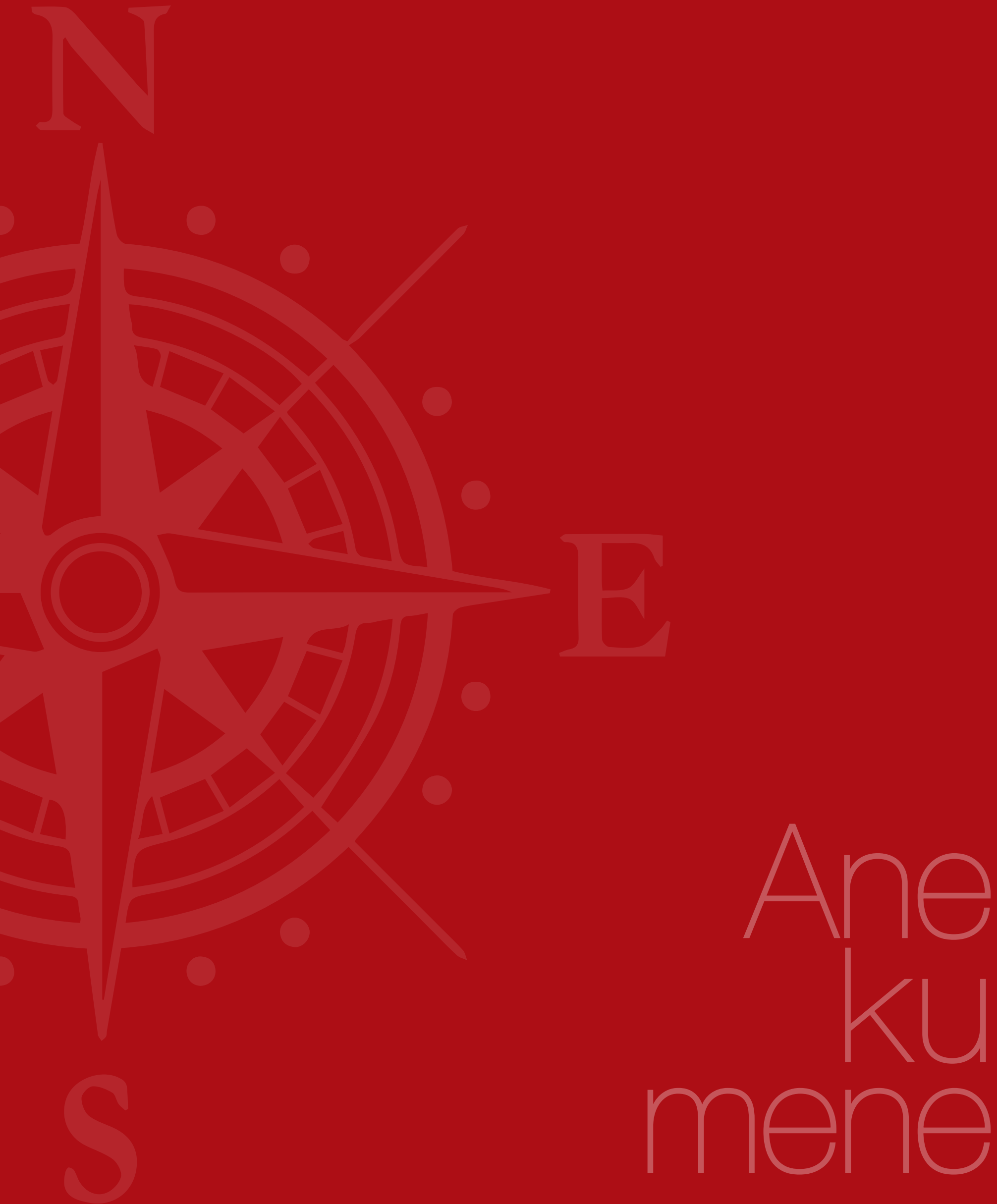


\section{Programa de Educación Geográfica de la Universidad de La Serena, Chile: avances de investigación en torno al desarrollo del pensamiento geográfico}

Geographic Education Program at the University of La Serena, Chile: Research Progress on the Development of Geographical Thinking

Programa de Educação Geográfica da Universidade da Serena, Chile: avanços de investigação em torno do desenvolvimento do pensamento geográfico

Fabián Araya Palacios*

\section{Resumen}

El trabajo presenta los avances de la línea de investigación en torno al desarrollo del pensamiento geográfico, ejecutada por el Programa de Educación Geográfica de la Universidad de La Serena, Chile. El artículo se organiza en dos secciones complementarias: en primer lugar, se contextualiza el programa de educación geográfica y sus objetivos más relevantes; en segundo lugar, se presentan los resultados de una investigación finalizada en Mayo de 2016, relacionada con la importancia de las estrategias didácticas utilizadas por los docentes de la región de Coquimbo, con la finalidad de propiciar el desarrollo de habilidades de pensamiento geográfico en estudiantes de educación media. Se espera, de esta manera, contribuir con la difusión de las líneas y proyectos de investigación desarrollados por integrantes de la Redladgeo, a través de la sección de informes y avances de investigación de la revista Anekumene.

\section{Palabras clave}

Pensamiento geográfico; educación geográfica; estrategias didácticas; habilidades de pensamiento

* Profesor titular de la Universidad de La Serena, Chile. El autor agradece al Proyecto de Mejoramiento Institucional (PMI-FIP-ULS N ${ }^{\circ}$ 1501) y al Proyecto IPA N 1641 de la Dirección de Investigación y Desarrollo de la Universidad de La Serena (DIDULS) por el apoyo brindado para la elaboración del presente trabajo. 
Programa de Educación Geográfica de la Universidad de La Serena, Chile: avances de investigación en torno al desarrollo del pensamiento geográfico Fabián Araya Palacios /

\section{Keywords Abstract}

eographical thinking, geographical education, teaching strategies, thinking skills.

\section{Palavras-chave}

Pensamento geográfico, educação geográfica, estratégias didáticas, habilidades de pensamento.

\section{Resumo}

The paper present the progress of the research on the development of geographical thinking, developed by geographical education program at the University of La Serena, Chile. It is organized into two complementary sections. First, the geographical education program and its most important objectives is contextualized. Second, the results of a completed research are presented in May 2016, related to the importance of the teaching strategies used by teachers in the Region of Coquimbo, in order to promote the development of skills of geographic thinking in high school students. It is expected, thus contributing to the spread of lines and research projects carried out by members of the Redladgeo, through progress reports and section research Anekumene journal.

O trabalho apresenta os avanços da linha de investigação em torno do desenvolvimento do pensamento geográfico, desenvolvida pelo Programa de Educação Geográfica da Universidade da Serena, Chile. Organiza-se em duas seções complementares. Em lugar primeiro, se contextualiza o Programa de Educação Geográfica e seus objetivos mais relevantes. Em lugar segundo, apresentam-se os resultados de uma investigação finalizada em Maio de 2016, relacionada com a importância das estratégias didáticas utilizadas pelos docentes da Região de Coquimbo, com a finalidade de propiciar o desenvolvimento de habilidades de pensamento geográfico em estudantes em média Educação. Espera-se, desta maneira, contribuir com a difusão de linhas e projetos de investigação desenvolvidos por Integrantes da Redladgeo, através da Seção de relatórios e avanços de Investigação da Revista Anekumene. 


\section{Introducción}

La educación geográfica, entre sus propósitos principales, debe propiciar que la relación ser humano y medioambiente se desarrolle sobre la base de una perspectiva integrada y sustentable del espacio geográfico (GENIP, 2012; IGU, 2016). Para lograr este propósito, se requieren profundos cambios en el estilo de vida y mayores conocimientos que promuevan la conciencia pública ambiental, la participación ciudadana y el desarrollo del pensamiento geográfico, a través de habilidades que permitan tomar decisiones en temas relacionados con el medioambiente y su conservación.

El pensamiento geográfico corresponde a un conjunto de relaciones sociales y naturales cuyo objetivo persigue que los alumnos integren la información adquirida, básicamente a través de los sentidos, en una estructura de conocimiento que tenga pertinencia para ellos (Amestoy, 2002). Actualmente, en el contexto cultural anglosajón, el concepto desarrollado para definir y profundizar este proceso se denomina Spatial Thinking. Ello implica investigar sobre diversas modalidades y estrategias para desarrollar el pensamiento espacial, desde la perspectiva social, didáctica y curricular (Gersmehl, 2008; Butt, 2011; Lee y Bednarz, 2012; Stuart, Bednarz, Gersmehl, Kolvoord y Uttal, 2013) ${ }^{1}$.

De acuerdo a la literatura especializada, la educación geográfica no ha logrado desarrollar cabalmente en los estudiantes un claro pensamiento geográfico y un conjunto de habilidades de pensamiento superior, que les permita comprender sistémicamente el espacio y las relaciones sociedad-naturaleza, desde el punto de vista del desarrollo sustentable (Souto, 2012). En consideración con lo anterior, el Programa de Educación Geográfica de la Universidad de la Serena ha impulsado una línea de investigación en torno al desarrollo del pensamiento geográfico, que se ha materializado en diversos proyectos de investigación y trabajos de postdoctorado. Ello ha permitido profundizar el tema de estudio y organizar un equipo de investigación interesado en desarrollar estrategias didácticas y metodológicas que permitan el desarrollo de habilidades de pensamiento geográfico con estudiantes de enseñanza básica y media de la región de Coquimbo.

\section{Programa de Educación Geográfica}

Actualmente, el Programa de Educación Geográfica se encuentra integrado por Sandra Álvarez Barahona, María Leonor Ascui Barth, Silvana Núñez Thompson y Fabián Araya Palacios. El programa se ha conformado como resultado de una iniciativa académica de profesores y profesoras de la carrera de Pedagogía en Historia y Geografía de la Universidad de La

1 Entre el 13 y el 15 de Abril del año 2015, se realizó una conferencia en el Instituto de Educación de la Universidad de Londres, titulada The Power of Geograpbical Thinking. En ella se analizó el concepto de pensamiento geográfico y se plantearon diferentes alternativas para su desarrollo en diversos países y culturas. Hubo consenso, entre los participantes, en torno a la importancia de este tema para la educación geográfica contemporánea (Brooks, 2015).
Serena. Surgió como una forma concreta de establecer vínculos al interior de la carrera entre la formación pedagógica, metodológica y de especialidad, y también como una manera de vincularse con el medio educativo externo a nivel regional. Su quehacer se ha visto enriquecido gracias al apoyo y la colaboración de autoridades universitarias (vicerrector académico, directora de docencia, etc.), profesores y estudiantes, quienes, a través de una red de trabajo colaborativo, tanto de Historia como de Geografía, han permitido un crecimiento sostenido del programa y un compromiso real y efectivo con el presente y el futuro de la educación geográfica chilena (ver fotografía 1).

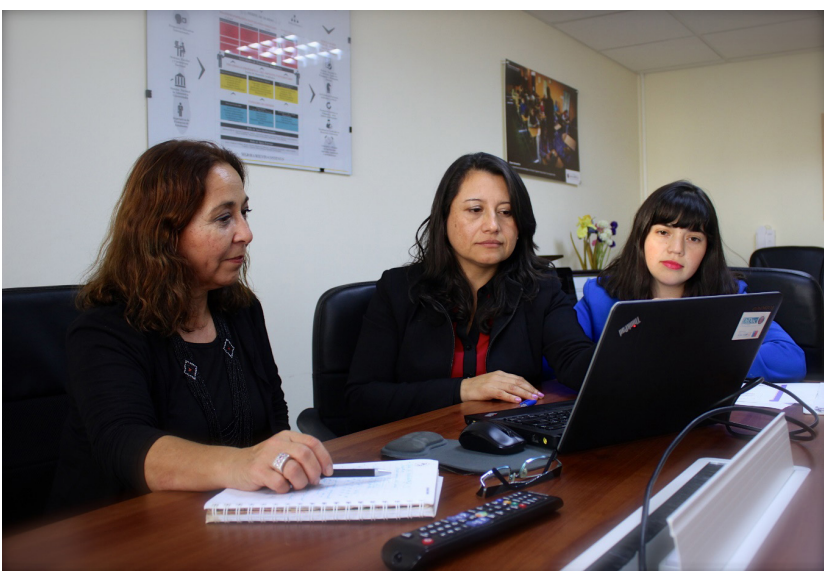

Fotografía 1. Integrantes del Programa de Educación Geográfica. Fuente: tomada por el autor.

El programa corresponde a una estructura académica funcional, orientada al estudio específico del proceso de enseñanza-aprendizaje de la geografía en el contexto de la educación básica, media y superior. A través de los resultados de estos estudios, se espera enriquecer la docencia, la investigación y la extensión de los académicos incorporados en el programa, de acuerdo a los desafíos que presenta el aprendizaje de los alumnos en el ámbito de la educación del siglo Xxı. El Programa de Educación Geográfica se ha planteado los siguientes objetivos:

\section{Objetivos generales}

- Propender hacia la formación inicial y permanente del docente de geografía como profesional reflexivo y autocrítico, capaz de tomar decisiones didácticas y metodológicas pertinentes a los diferentes ámbitos del quehacer educativo en los cuales le corresponda actuar.

- Potenciar líneas de investigación y desarrollo sobre aspectos relacionados con la didáctica de la geografía, que contribuyan a una profesionalización y perfeccionamiento continuo del docente de Ciencias Sociales. 


\section{Objetivos específicos}

- Promover el fortalecimiento de la capacidad de gestión académica del programa, a través de una permanente interrelación con las entidades públicas y privadas vinculadas con la temática a nivel regional, nacional e internacional.

- Contribuir al mejoramiento del proceso didáctico y metodológico en geografía desde la perspectiva de la renovación curricular, la incorporación de nuevas tecnologías de la información y comunicación (NTIC), y la optimización de las prácticas pedagógicas a nivel de aula.

- Desarrollar un proceso de apoyo, perfeccionamiento y actualización destinado a los docentes en ejercicio a través de jornadas, seminarios, generación de recursos didácticos y asesorías específicas en el ámbito de la didáctica y la metodología de la geografía.

- Incentivar la formulación y el desarrollo de diplomados, postítulos o posgrados en el ámbito de la didáctica y metodología de la geografía en permanente vinculación con las áreas de especialidad.

\section{Línea de investigación}

Corresponde a una iniciativa académica que se propone realizar aportes desde el punto de vista didáctico y metodológico para el desarrollo del pensamiento geográfico y el mejoramiento de la enseñanza-aprendizaje de la geografía. El programa elabora y ejecuta proyectos de investigación y de innovación en el ámbito de la didáctica y la práctica de esta disciplina. En los últimos años, los integrantes del programa de educación geográfica han ejecutado proyectos de investigación, financiados por el Fondo Nacional de Ciencia y Tecnología (Fondecyt) y la Dirección de Investigación y Desarrollo de la Universidad de La Serena (Diduls), tendientes al desarrollo del pensamiento geográfico. Una de las líneas de investigación más relevantes se ha denominado: Didáctica para el desarrollo del pensamiento geográfico. A través de ella, se estudia el vínculo entre los procesos didácticos, el pensamiento de los estudiantiles y la comprensión de las relaciones e interdependencias multidimensionales que ocurren en el espacio geográfico.

Se presenta, a continuación, los resultados del proyecto de investigación "Importancia de las estrategias didácticas para el desarrollo del pensamiento espacial sistémico: percepción de los docentes de Historia, Geografía y Ciencias Sociales de la Región de Coquimbo", financiado por la Dirección de Investigación y Desarrollo de la Universidad de La Serena (código Diduls Regular PR 14412), concluido en mayo de 2016. Este proyecto está relacionado con la relevancia de las estrategias didácticas utilizadas por los docentes de la región de Coquimbo, con la finalidad de contribuir al desarrollo del pensamiento geográfico en los estudiantes de educación media o secundaria.

\section{Síntesis del proyecto Diuls Regular PR 14412}

Título: Importancia de las estrategias didácticas para el desarrollo del pensamiento geográfico sistémico en los docentes de Historia, Geografía y Ciencias Sociales de la región de Coquimbo.

\section{Presentación}

Las nuevas tendencias de la enseñanza de la geografía, focalizadas en desarrollar el pensamiento geográfico con un enfoque más integral y sistémico, no se han materializado en Chile con los estudiantes de educación primaria y secundaria.

Además de identificar las dimensiones ambientales, sociales, económicas, políticas, culturales y tecnológicas que inciden en la vida cotidiana de los alumnos, es indispensable su comprensión de manera integrada. Para ello, el alumnado no debe ser visto como un ser pasivo, sino como agente cognoscente, cuyo aprendizaje debe estar basado en la construcción permanente del conocimiento. De allí, la importancia que presentan las habilidades cognitivas sobre las cuales se construye el conocimiento y su relación con la realidad geográfica actual.

Ante esta problemática, se procedió a elaborar un proyecto de investigación con el propósito de analizar las experiencias y estrategias utilizadas por los docentes de la región de Coquimbo (Chile) en torno al desarrollo del pensamiento geográfico sistémico, con sus estudiantes de educación primaria y secundaria. Para el desarrollo del proyecto se elaboró una encuesta aplicada a una muestra de 37 docentes (ver fotografía 2). La premisa de la investigación se fundamentó en que no basta con estudiar de manera aislada cada uno de los distintos objetos que conforman el espacio geográfico, sino que, por el contrario, resulta relevante comprender las interconexiones o interrelaciones que surgen entre los distintos elementos de un sistema espacial (Fernández, 2007).

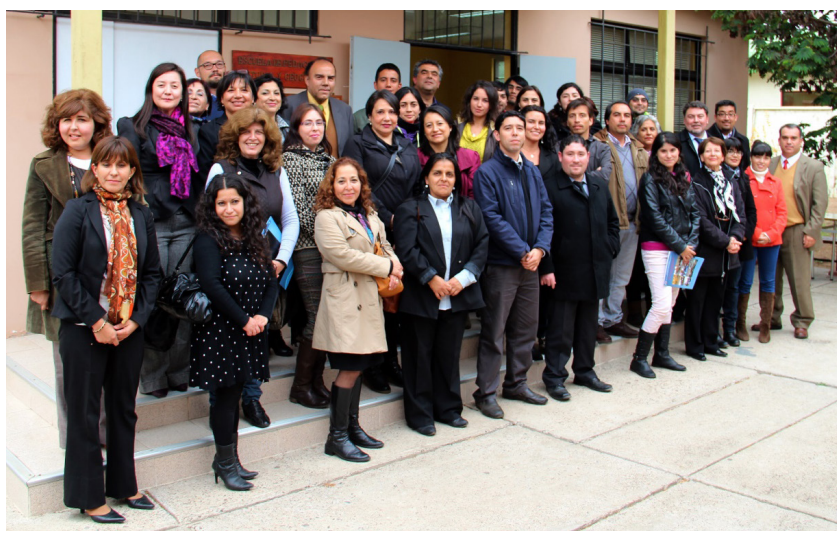

Fotografía 2. Docentes de Historia y Geografía en talleres didácticos. Fuente: tomada por el autor. 
El problema de investigación se planteó de la siguiente forma: ¿Cuáles son las estrategias didácticas utilizadas por los docentes de la región de Coquimbo para desarrollar con sus alumnos el pensamiento geográfico sistémico? La hipótesis de trabajo fue la siguiente: los docentes utilizan diferentes estrategias didácticas para el desarrollo del pensamiento geográfico, de acuerdo a su formación inicial, su experiencia docente y sus características contextuales.

La investigación, parte de la premisa de que una forma concreta de colaborar con el desarrollo del pensamiento geográfico consiste en actuar desde la educación geográfica, específicamente desde la didáctica de la geografía. Ello con la finalidad de elaborar propuestas didácticas que consideren el entorno local, las experiencias cotidianas de los alumnos y que, a través de un enfoque sistémico, contribuyan a que las nuevas generaciones se formen para una sociedad más compleja e interrelacionada.

\section{Desarrollo del pensamiento geográfico sistémico}

Un elemento central de la teoría constructivista del aprendizaje, es que este ocurre cuando un estudiante se ocupa de un problema que debe resolver. Según esta teoría, el aprendizaje se produce solo cuando los estudiantes hacen un esfuerzo para construir su propia comprensión de una situación problemática. Sin embargo, el esfuerzo del alumno se presenta principalmente en torno a las tareas de aprendizaje significativas; es decir, que aunque los profesores soliciten a los estudiantes la realización de trabajos escolares utilizando varios incentivos, el constructivismo sostiene que la comprensión profunda solo se produce cuando los partícipes se encuentran motivados a desarrollar esfuerzos, ya que se sienten involucrados y sus acciones dan sentido a su experiencia de la realidad.

El rol de la escuela en el desarrollo de las habilidades de pensamiento geográfico es muy relevante por cuanto prepara a los estudiantes para actuar en un mundo de movilidad local y global, en el cual las aptitudes de compresión de las representaciones simbólico-espaciales les permiten interactuar y comprender las relaciones dinámicas y las diferentes transformaciones de los elementos que componen los sistemas urbanos e, incluso, los espacios más reducidos. Por lo anterior, las habilidades que componen el pensamiento geográfico son imprescindibles en las actuaciones cotidianas, tales como desplazarnos por lugares conocidos o desconocidos, jugar al ajedrez o simplemente organizar o comprender la información de esquemas o diagramas.

Se presenta, a continuación, una propuesta para el desarrollo del pensamiento geográfico sistémico. A través de ella, se plantea un proceso gradual para desarrollar habilidades cognitivas que permita a las personas relacionarse con el entorno de manera sistémica, con la finalidad de adquirir, paulatinamente, una visión integrada del espacio geográfico (ver figura 1).

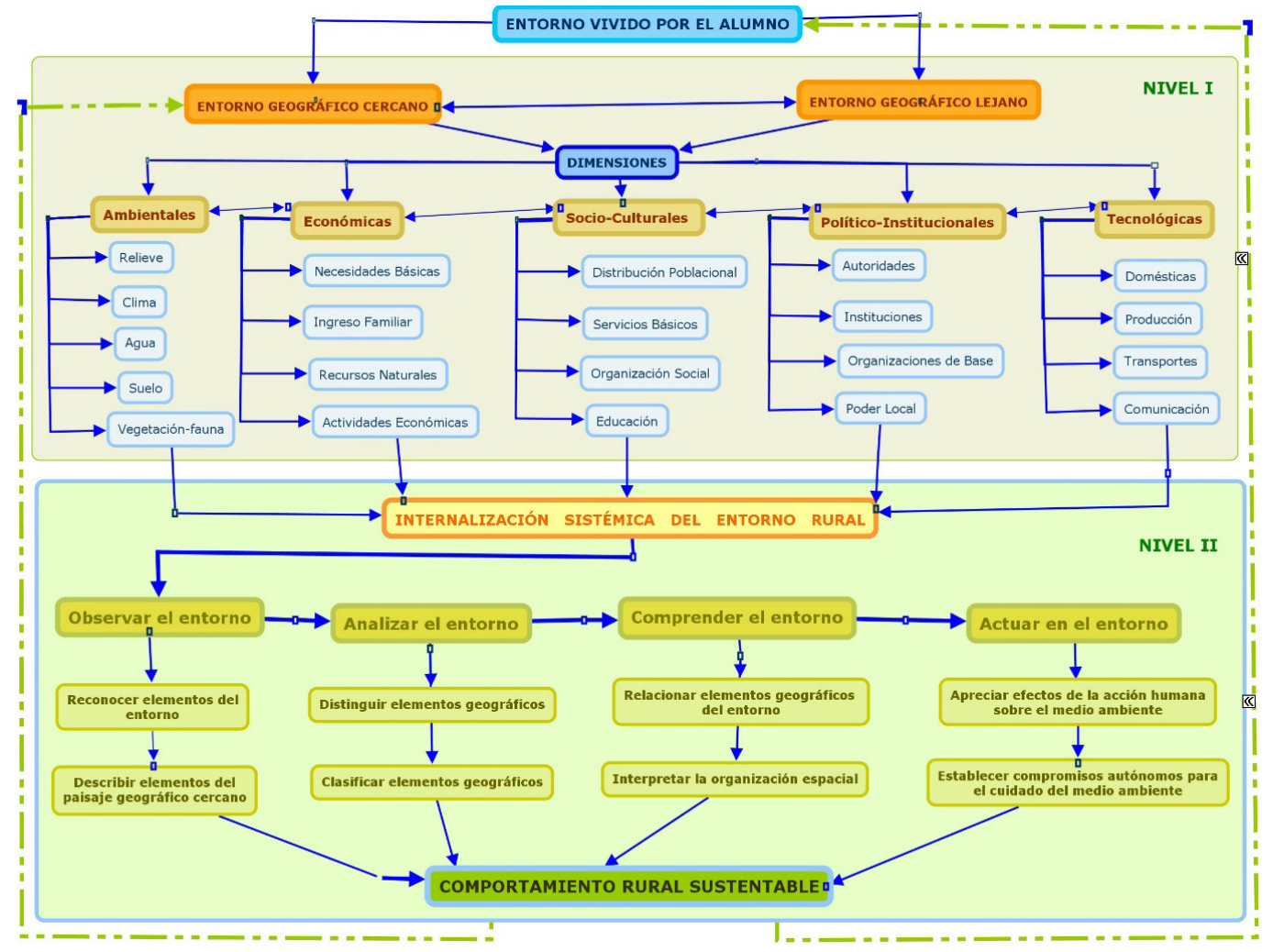

Figura 1. Propuesta para el desarrollo del pensamiento espacial sistémico.

Fuente: Araya, 2010, p 89 
El propósito de la propuesta didáctica es desarrollar, por parte de los alumnos, un pensamiento geográfico a través de una internalización sistémica del entorno geográfico. Para ello, se proponen cuatro habilidades cognitivas que se describen a continuación.

Observación del entorno: la observación constituye una fase necesaria para reconocer, identificar y localizar los elementos geográficos del entorno. Para desarrollar esta capacidad, necesaria para la formación de un pensamiento sistémico, es indispensable tener claros los objetivos de la observación. En vez de componentes geográficos aislados, se propone la observación, el reconocimiento y la descripción de elementos integrados del paisaje geográfico cercano.

Análisis del entorno: la segunda habilidad pretende que los alumnos desarrollen la capacidad cognitiva para clasificar y distinguir elementos del entorno. En lugar de reconocer factores aislados del espacio, en esta propuesta se enfatiza la clasificación de elementos del paisaje geográfico (Rodríguez, 2007).

Comprensión del entorno: esta habilidad pretende que los alumnos sean capaces de relacionar los elementos geográficos del entorno de acuerdo a las diversas dimensiones y componentes que han observado y analizado. El objetivo es llegar a interpretar la organización espacial relacionando, de manera integrada, las condiciones ambientales, económicas, socioculturales, político-instituciones y tecnológicas del medio, para apreciar los efectos de la acción humana sobre el ambiente (Rodríguez, 2007).

Actuación en el entorno: esta habilidad debería permitir evidenciar, a través de actitudes y acciones específicas realizadas por los estudiantes, el desarrollo del pensamiento geográfico para el cuidado del entorno cercano. Los indicadores para apreciar el cumplimiento de esta etapa son los siguientes:

- Apreciar efectos de la acción humana sobre el medioambiente: implica verificar si los alumnos y alumnas, de acuerdo a sus conocimientos previos, son capaces de apreciar integradamente los efectos negativos o positivos de la acción humana sobre el medioambiente. Es necesario que el modelo sea aplicado en forma gradual en diversos cursos.

- Establecimiento de compromisos autónomos para el cuidado del medioambiente: la propuesta pretende desarrollar la capacidad de los estudiantes para organizar sus conocimientos previos y sus nuevas experiencias a través de una visión sistémica de la realidad. En este sentido, sus acciones no tienen importancia por sí mismas sino por sus interacciones, y por los efectos que ocasiona el cumplimiento de sus compromisos en el resto del sistema geográfico.

La propuesta permite orientar el proceso didáctico desarrollado por los alumnos, guiados por un profesor o profesora hacia una visión integrada del espacio geográfico. Cada fase presenta actividades de aprendizaje diseñadas para desarrollar determinadas habilidades cognitivas en los educandos. A través de estas actividades se pretende avanzar, gradualmente, desde una visión fragmentada del espacio geográfico hasta una comprensión integrada o sistémica del entorno.

\section{Las prácticas educativas y los procesos cognitivos}

Se parte del supuesto que las habilidades que configuran el pensamiento geográfico, incorporadas en los objetivos curriculares de la enseñanza de la geografía, orienten la elección que hacen los docentes de las estrategias didácticas y las actividades de aula en procura de fortalecer dichas capacidades. Sin embargo, las prácticas educativas que realizan los docentes develan una falta de articulación entre las habilidades espaciales que se espera desarrollar y las actividades de aula que se planifican.

Si bien existen diversas prácticas de enseñanza compatibles con el desarrollo de habilidades para el pensamiento geográfico, algunas se vinculan de manera tangencial y no parecen estar dirigidas esencial o progresivamente al desarrollo de habilidades espaciales. Se encuentran centradas en la revisión de contenidos y temas determinados que, en algunos casos, obstaculizan el despliegue de destrezas que permitan comprender el espacio geográfico de manera integrada.

Se presenta, a continuación, la tabla 1, en la cual, a modo de ejemplo, aparecen relacionadas las habilidades para el desarrollo del pensamiento espacial sistémico (DPES),, con las estrategias didácticas y las actividades empleadas por los docentes de aula, con la finalidad de desarrollar este tipo de pensamiento. 
Tabla 1. Práctica docente de aula: organización de estrategias y actividades para el desarrollo del pensamiento geográfico sistémico.

\begin{tabular}{|c|c|c|c|c|c|}
\hline \multirow{3}{*}{ 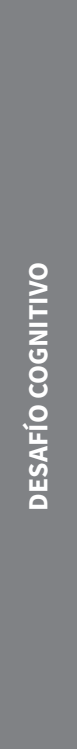 } & \multirow{2}{*}{ 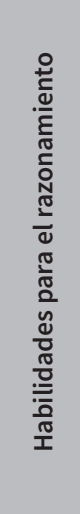 } & \multirow{2}{*}{ 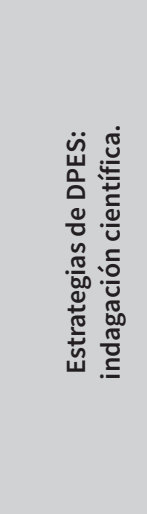 } & $\begin{array}{l}\text { Los estudiantes justifican y explican } \\
\text { su razonamiento o realizan debates } \\
\text { en torno a problemáticas ambientales } \\
\text { que afectan su entorno. }\end{array}$ & $\begin{array}{l}\text { Los estudiantes comprometen } \\
\text { actuaciones fundadas sobre } \\
\text { razonamientos informados y } \\
\text { posturas personales en relación } \\
\text { al cuidado del medioambiente y } \\
\text { los recursos naturales. }\end{array}$ & $\begin{array}{l}\text { Los estudiantes sugieren o } \\
\text { colaboran en la planificación } \\
\text { de un tema geográfico en } \\
\text { actividades de clase basadas } \\
\text { en los resultados de sus } \\
\text { indagaciones, entrevistas } \\
\text { o encuestas, para ofrecer } \\
\text { soluciones a problemas locales. }\end{array}$ \\
\hline & & & $\begin{array}{l}\text { Los estudiantes analizan datos } \\
\text { espaciales asociados a las estadísticas } \\
\text { y comunican el conocimiento } \\
\text { producto de la interpretación y su } \\
\text { relación con distintas fuentes de } \\
\text { información. }\end{array}$ & $\begin{array}{l}\text { Los estudiantes explican su } \\
\text { pensamiento en relación a una } \\
\text { serie de factores geográficos que } \\
\text { originan un grave problema de } \\
\text { sustentabilidad urbana. }\end{array}$ & $\begin{array}{l}\text { Los estudiantes escriben un } \\
\text { ensayo o una evaluación con } \\
\text { respecto a su propio trabajo } \\
\text { desarrollado en una visita a una } \\
\text { zona agrícola. }\end{array}$ \\
\hline & 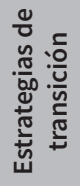 & 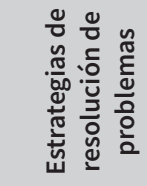 & $\begin{array}{l}\text { Los estudiantes trabajan en la } \\
\text { explicación de problemas ambientales } \\
\text { que afectan su entorno y que } \\
\text { presentan soluciones complejas. }\end{array}$ & $\begin{array}{l}\text { Los estudiantes plantean } \\
\text { problemas de acuerdo a las } \\
\text { observaciones realizadas } \\
\text { durante una salida de campo. }\end{array}$ & $\begin{array}{l}\text { Los estudiantes desarrollan } \\
\text { procedimientos para resolver } \\
\text { problemas espaciales complejos } \\
\text { y discuten sus resultados. }\end{array}$ \\
\hline 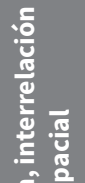 & 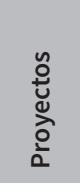 & \multicolumn{2}{|c|}{$\begin{array}{l}\text { Los estudiantes presentan su trabajo ante una audiencia, } \\
\text { extrapolando habilidades espaciales en la comprensión } \\
\text { de la biodiversidad y sus proyecciones energéticas. }\end{array}$} & \multicolumn{2}{|c|}{$\begin{array}{l}\text { Los estudiantes diseñan mapas dinámicos y de proceso, empleando } \\
\text { distintas fuentes de información y proyecciones sobre problemáticas } \\
\text { reales del espacio geográfico. }\end{array}$} \\
\hline 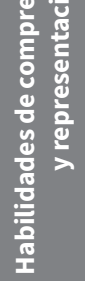 & 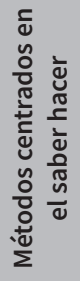 & \multicolumn{2}{|c|}{$\begin{array}{l}\text { Los estudiantes trabajan en pequeños grupos para } \\
\text { completar una tarea asignada por el docente. }\end{array}$} & \multicolumn{2}{|c|}{$\begin{array}{l}\text { Los estudiantes trabajan en grupo para interpretar mapas, analizar las } \\
\text { características de las proyecciones geográficas e interpretar patrones } \\
\text { de distribución de la población. }\end{array}$} \\
\hline 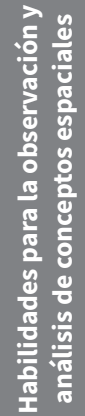 & 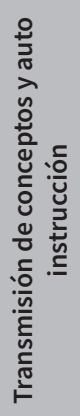 & \multicolumn{2}{|c|}{$\begin{array}{l}\text { Los estudiantes desarrollan una presentación } \\
\text { de geografía frente al grupo completo. }\end{array}$} & \multicolumn{2}{|c|}{$\begin{array}{l}\text { Los estudiantes responden individualmente } \\
\text { las preguntas a partir de láminas o textos. }\end{array}$} \\
\hline
\end{tabular}

Fuente: Ravitz, Becker y Wong, 2000. Adaptación del autor. 


\section{Materiales y métodos}

Se presentan, a continuación, los aspectos metodológicos considerados en la investigación:

Tipo de investigación: desde los fines extrínsecos o externos, el trabajo corresponde a una investigación de tipo aplicada. Esto se debe a que se pretende contribuir con la descripción de las experiencias y estrategias didácticas concretas a desarrollar el pensamiento geográfico sistémico. Desde los fines intrínsecos o internos, la presente investigación es de carácter descriptivo, pues se pretende sistematizar la información relacionada con la aplicación de una encuesta semiestructura a los docentes de geografía.

Muestra: según el Grupo Chadule (1980), esta corresponde a un “[...] subconjunto tomado de una población" (p. 16). Considerando que la muestra contiene las características de los sujetos a estudiar y, de acuerdo con los objetivos de la investigación, se ha seleccionado una muestra de tipo intencional de carácter no probabilística. Esta se encuentra conformada por dos grupos de treinta y siete (37) docentes en total. Para efectos del análisis de datos, se segmentaron los grupos de muestra en distintos tramos, de acuerdo a los años de experiencia de los encuestados. I: docentes con experiencia inicial, con menos de cinco años de trabajo educativo; M: docentes con experiencia media, con más de cinco años pero menos de 10, y A: docentes entre 11 y 23 años de práctica educativa. De esta organización, solo cinco (5) se ubicaron en el tramo de experiencia inicial (en adelante I), quince (15) en el intervalo medio (en adelante M) y diez y siete (17) en el subconjunto avanzado (en adelante A).

Diseño de la investigación: para desarrollar la investigación se utilizó un diseño de carácter no experimental. Una de las características de este enfoque es que "los sujetos no son asignados al azar a los grupos, ni emparejados; sino que dichos grupos ya [se encuentran] formados antes del experimento, son grupos intactos" (Hernández, 1991, p. 173). Este diseño se utilizó para organizar el proceso de investigación de acuerdo a cada una de sus fases y etapas. Permitió sistematizar la información cuantitativa, producto de la aplicación de la encuesta, así como reunir e interpretar la información cualitativa surgida a medida que se desarrollaba el proceso de investigación. Para la recolección de los datos se empleó como instrumento la encuesta docente (ver anexo 1).

\section{Resultados}

Se presentan los resultados del estudio, los cuales están organizados, en primer lugar, respecto a las estrategias didácticas preferidas por los docentes; luego, de acuerdo a las actividades de aula que materializan dichas estrategias y, finalmente, a partir de las habilidades del pensamiento geográfico, que se pretende fortalecer.
En cuanto a las estrategias preferidas por los docentes se presentan los resultados ordenados, en primera instancia, considerando el género de los profesores y, luego, asumiendo el tramo de experiencia en la labor educativa. En el caso de las estrategias metodológicas preferidas para el desarrollo del pensamiento geográfico, es posible evidenciar que un $50 \%$ de los docentes de género masculino prefieren desarrollar estrategias orientadas a la resolución de problemas, mientras que las profesoras lo hacen en un 35\%. Es interesante reconocer la importancia que reviste el hecho de que los docentes favorezcan este tipo de estrategias. Ello conlleva el fortalecimiento de las habilidades complejas de razonamiento, puesto que permiten a los estudiantes desarrollar conexiones entre datos, contenidos y posturas personales que trascienden el manejo de contenidos geográficos conceptuales.

De acuerdo a los resultados sobre las habilidades del pensamiento geográfico que los docentes privilegian y orientan en sus prácticas pedagógicas de aula, la habilidad preferida por los profesores es la de observación con un $69 \%$ de aprobación. Finalmente, la preferencia predominante de los docentes para promover habilidades espaciales, de acuerdo a los años de experiencia, es la habilidad de observación para todos los tramos presentados. Cabe anotar, en contraste, el bajo porcentaje de educadores que privilegia la interpretación (3,4\% de aquellos con experiencia inicial). El segmento de experiencia docente con mayor diversidad en cuanto a la elección de habilidades fue el de experiencia mediana.

\section{Interpretación}

Los cambios ocurridos en los últimos años respecto a las prácticas asumidas en la enseñanza a menudo se centran en la creencia de que son mejores aquellas en las que los estudiantes trabajan activamente en proyectos y que suelen incluir grupos de trabajo colaborativo o un producto final específico. No obstante, en las prácticas que promueven los procesos de razonamiento para el desarrollo del pensamiento geográfico no basta con la concurrencia de este tipo de actividades, sino que, además, se hace imprescindible la orientación pedagógica para hacer que dichas labores sean cognitivamente desafiantes para los alumnos y que no sean solo amenas por la actividad que entrañan. Por ejemplo, son las preguntas que emergen en una salida a terreno y la mediación de los docentes las que logran que los estudiantes construyan relaciones y analicen críticamente los fenómenos que son producto de la comprensión, la búsqueda y la interpretación de la información.

En la consulta realizada a los docentes, existe un predominio por la actividad vinculada con las salidas de campo (70\%). Ello se explica por la importancia que los docentes le asignan a la habilidad de observación en la enseñanza de la disciplina geográfica (ver fotografía 3). 


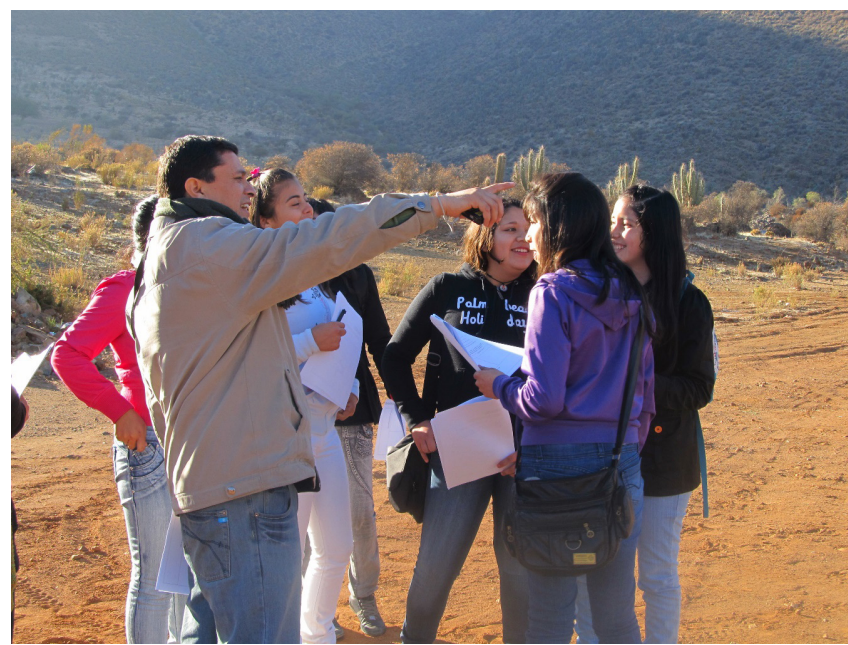

Fotografía 3. Docente de geografía en actividad de salida a terreno. Fuente: tomada por el autor.

\section{Discusión}

El pensamiento geográfico ha sido comprendido por el National Research Council (2006), como un conjunto de habilidades cognitivas que integran formas declarativas, habilidades de percepción del conocimiento espacial y algunas operaciones cognitivas, que se pueden utilizar para analizar, comprender, transformar y producir nuevas formas de conocimiento del espacio geográfico. Asimismo, señala que el conocimiento espacial entraña el aprendizaje y el uso combinado de tres elementos clave: los conceptos de espacio, los procesos de razonamiento y las herramientas de representación y relación entre componentes. Es innegable el rol que juega la mediación docente para el fortalecimiento de las habilidades espaciales en el aprendizaje de la geografía.

Se desprende de los resultados descritos en la sección anterior que la habilidad de observación es la preferida por los docentes para el desarrollo del pensamiento geográfico. Como señala Rodríguez (2007):

La observación es de vital importancia [...] porque implica una tarea de exploración con un propósito específico: descubrir características, rasgos, relaciones, posiciones que posibilitan resolver problemas de conocimiento. No es una tarea simple, ni pasiva, requiere un constante intercambio entre la mente del observador y el objeto observado: el observador interroga y el objeto responde. (p. 143).

En los resultados de este estudio, la preferencia por las habilidades de observación y en segundo término de comprensión, parece guiar, a su vez, la tendencia de los docentes por desarrollar actividades de salida a terreno. A partir de estas actividades de campo, se ve fortalecida, por ejemplo, la modalidad de aprendizaje espacial del aura, entendida como la zona de influencia de factores geográficos (tales como ríos, empresas, centrales nucleares, bases de misiles etc.) y la desregionalización, por cuanto las condiciones geográficas son extensibles a localidades adyacentes (Gersmehl y Gersmehl, 2007).

Los estudiantes, al identificar no solo los elementos que componen el espacio sino al comprender cómo, por ejemplo, los factores climáticos se relacionan con el tipo de hábitat, las condiciones económicas o culturales que comparten lugares cercanos y que explican su pertenencia a una región por su área de influencia, hacen de la salida a terreno una instancia de aprendizaje propicia para el desarrollo de la comprensión espacial sistémica, siempre y cuando esté mediada y dirigida a trascender la descripción del paisaje. En palabras de Rodríguez (2007):

Al describir se pinta, se dibuja a través de la palabra; es entonces una representación que involucra objetos, hechos, procesos reales, experiencias, vivencias, lo que se percibe, se recuerda, se siente, se piensa, se imagina, del macro/micro entorno donde el sujeto se desenvuelve. Es vital enfatizar, en el marco de la descripción la importancia de establecer las relaciones para comprender los procesos socio-espaciales que protagoniza el hombre. (p. 146).

Dentro de las estrategias didácticas preferidas por los docentes se encuentran la indagación científica y la resolución de problemas, en cuya fase inicial juega un rol importante la habilidad de observación. No obstante, el planteamiento más profundo de actividades que desarrollen aptitudes de razonamiento y actuación en el contexto del pensamiento geográfico, está enfocado hacia acciones de resolución de problemas basadas en el planteamiento de situaciones hipotéticas, que exigen de los alumnos una actitud activa y un esfuerzo por buscar sus propias respuestas.

Enseñar a resolver problemas fomenta en los alumnos la capacidad de aprender a aprender (habilidades meta-cognitivas). Los estudiantes necesitan adquirir facultades y estrategias que les permitan aprender por sí mismos nuevos conocimientos. En el contexto de los problemas ambientales, se espera sensibilizar a los educandos frente a los problemas sociales y medioambientales glocales que les permita identificar dificultades, buscar soluciones, hacer especulaciones o formular hipótesis. finalmente, establecer no solo una postura discursiva, sino un compromiso real con el entorno a partir de sus procesos de razonamiento.

A partir del análisis de los resultados de este estudio, se observa la necesidad de propiciar en el aula el uso de diversos lenguajes de representación espacial, no solo con la cartografía sino también con las estadísticas, cuyo uso fue prácticamente nulo. Si bien los docentes señalaron en el estudio, la disposición a utilizar materiales cartográficos, resulta importante enriquecer estas actividades con la incorporación de procesos espaciales que involucren habilidades de razonamiento. La importancia de esta habilidad recae en que: 
[...] es un proceso sistemático que se basa en la confrontación de rasgos y fenómenos análogos existentes en la biosfera, buscando entender su comportamiento de acuerdo con regularidades, identidades, correlaciones, distinciones y divergencias, es decir, cotejar in situ lo que se observa con otras realidades y tiene como efecto afinar la explicación con contundencia científica. (Rodríguez, 2007, p.147).

Finalmente, es necesario referirse a la baja predilección de los profesores por el desarrollo de la habilidad de actuación como parte de los objetivos que orientan las estrategias y actividades docentes. En este sentido, tanto las actividades de resolución de casos y las estrategias de indagación científica o aprendizaje basado en problemas parecen no estar orientadas al desarrollo de habilidades espaciales de razonamiento y actuación espacial, sino más bien enfocadas a desarrollar las aptitudes de observación, análisis y comprensión. Estos resultados plantean la imperiosa necesidad de encaminar los currículos escolares al desarrollo de actitudes ciudadanas responsables, comprometidas y sensibles a la problemática ambiental y social, formando estudiantes capaces de analizar, organizar hipótesis y ensayar propuestas de solución acordes con actuaciones más responsables con el entorno.

\section{Conclusiones}

De acuerdo a los resultados y a la discusión precedente, se presentan las principales conclusiones del estudio organizadas de acuerdo a los ejes temáticos desarrollados en el proyecto.

Relevancia de la educación geográfica: la educación geográfica constituye una disciplina educativa que posee una gran potencialidad para la formación de los ciudadanos del presente y del futuro. Uno de los propósitos más importantes de la educación geográfica es contribuir al desarrollo del pensamiento geográfico como un desafío disciplinario y pedagógico. Esta disciplina debe propiciar que la relación ser humano-medioambiente se desarrolle sobre la base de una perspectiva integrada del espacio. Para ello, el rol de la escuela en el desarrollo de las habilidades de pensamiento geográfico es muy relevante, por cuanto prepara a los estudiantes para actuar en un mundo interdependiente local y globalmente.
Relevancia de los proyectos de investigación: el trabajo de indagación de diferentes realidades culturales y geográficas permite el enriquecimiento recíproco y el avance en los grados de generalización de los resultados que constituyen la base del conocimiento científico. En este caso, el trabajo con docentes de historia y geografía permitió corroborar la hipótesis de que los profesionales de la educación utilizan diferentes estrategias didácticas para el desarrollo espacial sistémico, de acuerdo a su formación inicial, su experiencia docente y sus características culturales.

Desarrollo del pensamiento geográfico: el trabajo con los docentes permitió constatar que sus estrategias didácticas están orientadas fundamentalmente hacia la labor de observación y comprensión de conceptos espaciales y, asimismo, hacia las actividades de interrelación y representación. Como punto de refuerzo, cabe anotar que falta desarrollar entre los docentes las estrategias de razonamiento espacial. Ello conlleva a un desafío cognitivo necesario para avanzar a mayores niveles de integración y actuación en el espacio geográfico. De manera más específica, es posible evidenciar que los docentes prefieren desarrollar estrategias orientadas a la resolución de problemas. Las estrategias metodológicas preferidas para el fomento del pensamiento geográfico, de acuerdo a la experiencia docente, varían en relación al tramo en el que se encuentran en su práctica profesional. Con menos años de experiencia, las estrategias se focalizan en el aprendizaje basado en problemas y los métodos de indagación científica, mientras que los profesores con más de diez años de trabajo educativo emplean una variada gama de destrezas en su práctica docente. La habilidad de la observación es la preferida por los encuestados para el desarrollo del pensamiento geográfico y, en segundo término, prefieren la habilidad de comprensión. Resulta relevante la actividad de salida a terreno como la más recurrente al momento de seleccionar una estrategia idónea para el desarrollo del pensamiento geográfico.

Los resultados de la investigación proporcionan un gran estímulo para seguir indagando sobre las habilidades de desarrollo del pensamiento espacial en el contexto de la educación geográfica actual. Solamente a través de ciudadanos geográficamente informados podremos mantener la esperanza de tomar decisiones y actuar correctamente en un mundo cada vez más globalizado e interdependiente. 


\section{Anexos}

\section{Anexo 1. Encuesta docente}

Nombre:

\section{Ciudad:}

Nivel de desempeño (Básico, Medio, Universitario):

Años de experiencia en docencia:
Establecimiento donde se desempeña:

E-mail:

I. Para cada una de las preguntas marque tres alternativas numerándolas de $\mathbf{1}$ a 3 , según su grado de prioridad o preferencia. 1 es la opción más importante y 3 la menos importante.

1. Si Ud. se planteara como objetivo en su práctica docente el lograr en sus alumnos la comprensión integral de su entorno geográfico, ¿cuál de estas actividades de enseñanza utilizaría?

Salida a terreno
Búsqueda de información a través de Internet
Uso de cartografía temática
Uso de estadísticas
Resolución de casos

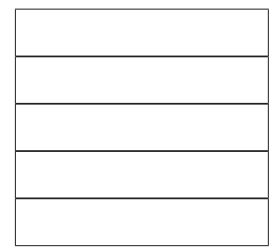

2. ¿Qué estrategias de aprendizaje Ud. considera relevantes implementar en el aula, para lograr en sus estudiantes un adecuado pensamiento geográfico sistémico?
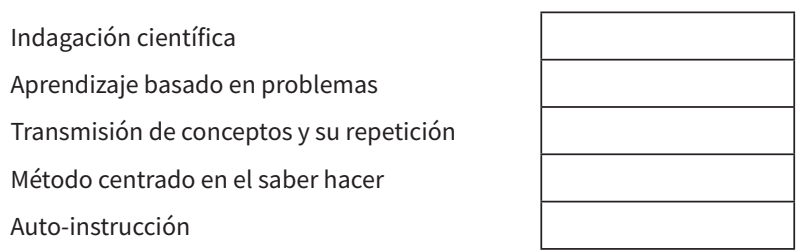

3. ¿Qué habilidades cognitivas considera Ud. que es trascendental desarrollar en sus estudiantes, para lograr aprendizajes significativos en torno al pensamiento espacial sistémico?

$$
\begin{aligned}
& \text { Observación } \\
& \text { Comprensión } \\
& \text { Análisis } \\
& \text { Interpretación } \\
& \text { Actuación }
\end{aligned}
$$

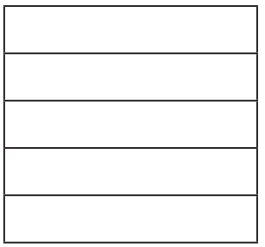

\section{Responda desde su punto de vista las siguientes preguntas. Fundamente su respuesta.}

1. ¿Qué aspectos relacionados con la enseñanza de la geografía adquiridos en su formación inicial geográfica, destacaría positivamente y cuáles no?

2. ¿El "desarrollo del pensamiento geográfico sistémico" está presente en el actual currículo de su país y con qué actividades didácticas se desarrolla en el aula? 
Programa de Educación Geográfica de la Universidad de La Serena, Chile: avances de investigación en torno al desarrollo del pensamiento geográfico Fabián Araya Palacios /

\section{Referencias}

Amestoy, M. (2002). La investigación sobre el desarrollo y la enseñanza de las habilidades de pensamiento. Revista Electrónica de Investigación Educativa, 4(1). Recuperado de: http://redie.uabc.mx/ redie/article/view/55.

Araya, F. (2010). Educación geográfica para la sustentabilidad. La Serena, Chile: Universidad de La Serena.

Brooks, C. (Abril de 2015). International differences in thinking geographically, and why it matters. En The Power of Geographical Thinking. Conferencia llevada a cabo en IGU - CEG. Londres, Inglaterra.

Butt, G. (Ed.). (2011). Geography, Education and the Future. London, Great Britain: Continuum International Publishing Group.

Fernández, M. (Coord.). (2007). Geografía y territorios en transformación. Nuevos temas para pensar la enseñanza. Buenos Aires, Argentina: Noveduc.

Geography Education National Implementation Project (GENIP). (2012). Geography for Life: National Geography Standards. Washington, Estados Unidos: National Council for Geographic Education.

Gersmehl, P. (2008). Teaching Geography. New York, Estados Unidos: The Guilford Press.

Gersmehl, P. y Gersmehl, C. (2007). Spatial Thinking by Young Children: Neurologic Evidence for Early Development and "Educability". Journal of Geography, 106(5), 181-191.

Grupo Chadule. (1980). Iniciación a los métodos estadísticos en geografía. Barcelona, España: Ariel.

Hernández, R. (1991). Metodología de la investigación. Bogotá, Colombia: McGraw-Hill.
International Geographical Union (IGU). (2016). Official website of the Commission on Geographical Education. Recuperado de: http:// www.igu-cge.org/charters.htm

Lee, J. y Bednarz, R. (2012). Components of Spatial Thinking: Evidence from a Spatial Thinking Ability Test. Journal of Geography, 111(1), 15-26.

National Research Council. (2006). Learning to Think Spatially. Washington, Estados Unidos: National Academies Press.

Ravitz, J., Becker, H., y Wong, Y. (2000). Constructivist-compatible beliefs and practices among U.S. teachers: teaching, learning, and computing. Center for Research on Information Technology and Organizations, Irvine, CA. Universidad de Minnesota. Recuperado de: http://citeseerx.ist.psu.edu/viewdoc/ download?doi=10.1.1.307.315\&rep=rep1\&type=pdf

Rodríguez, L. (2007). Una geografía escolar (in) visible. Desarrollo del pensamiento geográfico desde la construcción de conceptos geográficos. Bogotá, Colombia: Universidad Distrital Francisco José de Caldas.

Souto, X. (2012). O interesse da investigação na aprendizagem e didáctica da Geografia. En Vanzella, S., De Souza, L., y Copetti, H. (Coord.) Didática da Geografía: aportes teóricos e metodológicos. (pp. 63-84). São Paulo, Brasil: Xama.

Stuart, D., Bednarz, S., Gersmehl, P., Kolvoord, R., y Uttal, D. (2013). The People 's Guide to Spatial Thinking. Washington, Estados Unidos: National Council for Geographic Education. 